\title{
Checkerboard-Like Charge Ordering in Underdoped Cuprates
}

\author{
P. WRÓBEL* \\ Institute for Low Temperature and Structure Research \\ P.O. Box 1410, 50-950 Wrocław 2, Poland
}

\begin{abstract}
Recent scanning tunneling microscopy measurements which indicate the formation of two-dimensional density modulations at some doping levels in cuprates were reviewed. A model of hard-core bosons which represent bound hole pairs in the two-dimensional doped antiferromagnet was discussed in the context of these experimental results. By means of an exact numerical diagonalization it was shown that the Coulomb repulsion between bosons brings about the formation of charge modulations.
\end{abstract}

PACS numbers: 74.20.Mn, 71.10.Fd

\section{Introduction and overview of some experimental results}

Hole doping changes substantially the behavior of planar copper oxides. The phase diagram of these systems shows four main phases at zero temperature. Three of them, metallic, insulating, and superconducting are rather conventional. The fourth, the pseudogap phase is not very well understood, despite that it was found longer than a decade ago. The complexity of phenomena which occur in this phase prompts a suggestion that it contains an additional electronic order, for example a form of charge density wave (CDW). The scanning tunneling microscopy (STM) is a technique able to detect charge modulations, because it can measure local density of states (LDOS) at the surface of a material. A study in the magnetic field demonstrated regular LDOS modulations in the vicinity of vortex cores in $\mathrm{Bi}_{2} \mathrm{Sr}_{2} \mathrm{CaCu}_{2} \mathrm{O}_{8+\delta}$ (Bi-2212) compounds [1].

In our article we do not discuss phenomena which are induced by an external field. Here we concentrate on recent announcements which suggest that similar LDOS modulations are also generated when any external field is not applied. We start a short review of new experimental results with the discussion of low-temperature electronic structure studies in a lightly hole-doped copper oxide, $\mathrm{Ca}_{2-x} \mathrm{Na}_{x} \mathrm{CuO}_{2} \mathrm{Cl}_{2}$ (Na-CCOC) [2]. This system is suitable to discuss the evolution of electronic properties with doping. Its parent compound $\mathrm{Ca}_{2} \mathrm{CuO}_{2} \mathrm{Cl}_{2}$

*e-mail: p.wrobel@int.pan.wroc.pl 
is a canonical antiferromagnetic (AF) Mott insulator, which may be transformed by sodium substitution for calcium into an unconventional metallic phase and eventually into the superconducting state for $x \geq 0.1$. The energy resolved differential tunneling conductance, $g\left(\boldsymbol{r}, E=e V_{s}\right)$ obtained from STM experiments has been used to analyze charge modulations in a single copper-oxygen plane of the Na-CCOC system. This quantity is an indirect measure of energy dependent single particle $\operatorname{LDOS}(\boldsymbol{r}, E)$. Despite that Na-CCOC can be cleaved only between $\mathrm{CaCl}$ layers, it is believed that the properties of $g(\boldsymbol{r}, \boldsymbol{E})$ are determined by states in the $\mathrm{CuO}_{2}$ plane, because the $\mathrm{CaCl}$ layers are strongly insulating. Hoffman et al. performed their measurements for the doping level $0.08 \leq x \leq 0.12$. The spectra of conductance are strongly bias asymmetric, which may be attributed to particle-hole asymmetry of the system near Mott insulator transition (MIT). The maps of $g(\boldsymbol{r}, \boldsymbol{E})$ at constant $E=8,24$, and $48 \mathrm{meV}$ in the field of view $20 \mathrm{~nm} \times 20 \mathrm{~nm}$ show similar periodicities of modulations. The analysis of the Fourier transform (FT) of these spectra $g(\boldsymbol{q}, \boldsymbol{E})$ gives rise to the conclusion that among these modulations dominates a checkerboard-like $4 a_{0} \times 4 a_{0}$ structure, because $g(\boldsymbol{q}, \boldsymbol{E})$ is symmetric under rotations by $\pi / 2$ and shows intensity maxima for $\boldsymbol{q}=( \pm 1 / 4,0) 2 \pi / a_{0},(0, \pm 1 / 4) 2 \pi / a_{0}$. It seems that positions of these maxima do not disperse much with energy.

The question whether wave vectors, at which conductance maxima appear, disperse with energy is a central issue for the identification of charge order. Some time ago STM of Bi-2212 revealed weak, incommensurate, spatial modulations in the tunneling conductance [3]. Due to dispersion with energy of intensity maxima in Fourier transformed (FTed) conductance maps, these modulations were not attributed to charge order, but to quasiparticle interference effects. We will discuss now these results with some details. In the presence of impurities, elastic scattering mixes quasiparticles with different wave vectors $\boldsymbol{k}$ but with the energy is conserved. Therefore, these wave vectors are located on a contour of constant energy in $\boldsymbol{k}$ space. When scattering mixes states $\boldsymbol{k}_{1}$ and $\boldsymbol{k}_{2}$, an interference pattern with the wave vector $\boldsymbol{q}=\boldsymbol{k}_{2}-\boldsymbol{k}_{1}$ appears in the quasiparticle wave function and LDOS modulations with wavelength $2 \pi / q$ are formed. Those modulations can be observed by STM as modulations of the differential tunneling conductance. Due to lifetime effects quasiparticles are well defined only near the Fermi surface. The form of the Fermi surface in Bi-2212 has been identified by means of angle resolved photoemission spectroscopy (ARPES) [4-6]. The energy dispersion of quasiparticles in BCS superconductors (SCs), to which cuprates bear some similarity (and Bi-2212 among them), is gapped. It turns out that constant quasiparticle energy contours in that system have the shape of four bananas. Each of these bananas is symmetric with respect to one of the lines $k_{x}= \pm k_{y}$. The ends of those "bananas" are directed at points $( \pm \pi, 0),(0, \pm \pi)$. Each of four bananas is located in different quarters of the square first Brillouin zone. An important experimental observation is that their size increases when the quasiparticle energy increases. 
The scattering is effective when the density of states for both wave vectors initial $\boldsymbol{k}_{1}$ and final $\boldsymbol{k}_{2}$ is high. The density of states is high in regions where the curvature of constant quasiparticle energy contour is small. In the case of bananalike contours, their ends have small curvature. The wave vectors which connect nearest ends of two different bananas are parallel to zone boundaries. The length of these vectors decreases when the size of bananas and the quasiparticle energy increase. The wave vectors which connect ends of the same banana are parallel to zone diagonals. The length of these vectors increases when the size of bananas and the quasiparticle energy increase. The changes with quasiparticle energy of wave vectors which connect high density regions at constant energy contours have been measured by means of ARPES. The changes with energy of wave vectors at which intensity maxima appear in the FT conductance maps have been measured by means of scanning tunneling spectroscopy (STS). Since they are in almost quantitative agreement, a conclusion can be drawn that density modulations observed in the STM experiments reported in $[3,7]$ are driven by interference effects.

The above discussion of different interpretation which may have modulations observed in STM experiments on the surface of planar copper oxides, prompts to ask the question, whether the suggestion that an electronic crystal is formed in these systems, is valid at all? It seems that the answer to that question was given in a recent paper published by the Davis group [8], the results of which we mostly discuss in this short review. This group has analyzed Bi-2212 at doping levels in the range between $\delta=10 \%$ and $\delta=20 \%$. In the FT conductance maps, maxima at $\boldsymbol{q}=( \pm 2 \pi, 0),(0, \pm 2 \pi)$ have been observed. These maxima seem to be caused by umklapp scattering of the Bogolyubov quasiparticles between $\boldsymbol{k}=( \pm \pi, 0),(0, \pm \pi)$. They disappear at lower doping when the Fermi surface shrinks in the antinodal regions and takes form of four arcs which do not touch zone boundaries. In order to analyze the behavior of conductance maps at low doping the Davis group excited regions where the gap $\Delta$ is smaller than $65 \mathrm{meV}$. In the FTs of masked maps, which have been obtained by neglecting regions where the gap value is high, the intensity maxima which disperse with energy can be seen. Their appearance may be attributed to interference effects in the same way as it has been done in the earlier STM analyses of Bi-2212 compounds. The reason of this interpretation is the above mentioned observation of dispersion of their positions with energy. The dispersion of maxima positions is not seen, when in the conductance map at low doping level the regions with low values of the gap below $65 \mathrm{meV}$ are neglected, before the FT is applied. The nondispersive maxima which are only seen at higher energies are located at positions $\left( \pm 4.5 / a_{0}, 0\right)$ and $\left(0, \pm 4.5 / a_{0}\right)$. Additionally, different dispersive maxima are also formed in this case at lower energies, which correspond to lower bias voltage. This second class of maxima can again be attributed to interference effects. The observations made by the Davis group can be summarized as follows. Quasiparticle interference in Bi-2212 occurs between states located at tips of banana-like constant quasiparticle energy contours. These 
quasiparticles seem to be Bogolyubov-like because particle-hole symmetry may be observed. At lower doping quasiparticles in antinodal regions disappear, because Fermi arcs shrink. The destruction of antinodal quasiparticles coincides with appearance of regions with high value of gap energy. The notion of a pseudogap is used in this case, because sharp peaks in conductance spectra are absent. In the pseudogap regions non-dispersive modulations of LDOS are observed at high energies. They may be interpreted as unusual charge order. Since these modulations are observed in the single particle density only at high energies, it is tempting to attribute them to ordering of bound pairs. Low energy dispersive features which are observed in all cases indicate the existence of the Bogolyubov quasiparticles both in pseudogapped and gapped regions.

\section{A model of charge ordering in underdoped cuprates}

Now, after we have discussed experimental results, which indicate that charge-ordering takes place in some weakly doped cuprates, we will try to check if this phenomenon can be understood in the framework of an effective model based on the $t-J$ model $(t J \mathrm{M})$. The underlying assumption which we make during the construction of the effective model, is that at least short range AF correlations exist in the system. This assumption seems to be valid for underdoped cuprates. More information about the model and about its justification may be found in an earlier publication [9]. For the purpose of this paper it is sufficient to mention that

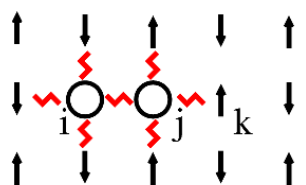

(a)

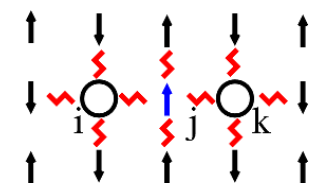

(b)

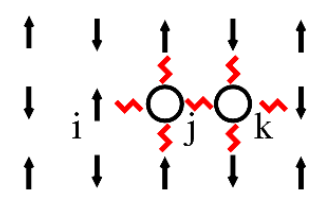

(c)

Fig. 1. The process giving rise to hopping of spin bipolarons which in the effective Hamiltonian $H_{\text {eff }}$ are represented by hard core bosons.

the effective model is defined in terms of hard-core bosons representing spin bipolarons or, in different words, bound hole pairs. The kinetic part of that originates from a process depicted in Fig. 1

$$
H_{\text {eff }}^{(k)}=-\tau \sum_{(i, j, m) \in \mathcal{A}} b_{\langle i, j\rangle}^{\dagger} b_{\langle i, m\rangle} .
$$

The operators $b_{\langle i, j\rangle}$ refer to spin bipolarons and $\tau$ is a function of parameters $t$ and $J$ which appear in the $t J \mathrm{M}$. The set $\mathcal{A}$ contains trios of points which belong to the square lattice on which the $t J \mathrm{M}$ is defined. In each such trio $(i, j, m) i$ is a nearest neighbor of $j$ and $m . j$ and $m$ are not identical. The order in which they appear in $(i, j, m)$ is relevant. Since the spin bipolaron created at a bond between a pair of nearest-neighbour sites $\langle i, j\rangle$ is combination of states obtained by hopping of holes which have been initially created in the AF background on these sites, another 
spin bipolaron cannot occupy a bond which shares its end-point with the bond between $i$ and $j$. It is also clear that, for the same reason, no more than one spin bipolaron can occupy a single bond. Thus spin bipolarons are hard-core bosons (HCBs). These HCBs interact via screened Coulomb interaction. We assume that the unscreened Coulomb energy of two holes located at the distance of one lattice spacing is $V=t$, which is about $0.5 \mathrm{eV}$. Since HCBs are bound hole pairs, in the formula which represents the electrostatic interaction between them, the additional factor 4 will appear. We also assume that the screening length is about one lattice distance for the doping level $1 / 8$ and even shorter for higher doping levels. A simple analysis based on the application of the Poisson formula to layered system indicates that this assumption is reasonable. In addition, it is known that the coherence length in cuprates is short which indicates that the screening of the Coulomb repulsion is substantial. By collecting the above mentioned assumptions about the nature of the Coulomb interaction between spin bipolarons we may write the interaction term as

$$
H_{\mathrm{eff}}^{(\mathrm{C})}=-4 V \sum_{\langle\langle i, j\rangle,\langle m, n\rangle\rangle} \frac{\mathrm{e}^{-k_{0} r_{\langle i, j\rangle,\langle m, n\rangle}}}{r_{\langle i, j\rangle,\langle m, n\rangle}} n_{\langle i, j\rangle} n_{\langle i, m\rangle},
$$

where $1 / k_{0}$ is the screening length, $r_{\langle i, j\rangle,\langle m, n\rangle}$ denotes the distance between the centers of bonds $\langle i, j\rangle$ and $\langle m, n\rangle$ and $n_{\langle i, j\rangle}$ represents the HCB density at the bond $\langle i, j\rangle$.

\section{Results of numerical analysis and summary}

For the purpose of this paper, we consider a finite cluster in the checkerboard lattice. This cluster consists of 72 sites which represent the same number of bonds in $6 \times 6$ cluster of sites which form the square lattice on which the initial $t J \mathrm{M}$ is defined. We analyze $4 \mathrm{HCBs}$ in the checkerboard-like cluster. This number corresponds to 8 holes in the $t J \mathrm{M}$ on the $6 \times 6$ cluster. Thus, the doping level is $\delta=2 / 9$ in this case. This doping level is higher than doping levels at which charge ordering and pseudogap phase are observed in cuprates. In addition, it seems that for such a high value of the doping parameter the phenomenology of spin polarons may be not applicable, because the AF correlation length is very short in this region of the phase diagram for cuprates. Nevertheless, we hope to get some insight into the mechanism of charge ordering in underdoped cuprates, by analyzing this process in the framework of our model for a slightly unphysical parameter range. The results of numerical calculation for lower doping levels, which require much more extensive computational time, because cluster should be bigger in this case, will be presented elsewhere.

In order to check if the system of HCBs representing spin bipolarons forms a charge ordered state we calculate the density autocorrelation function $g(\boldsymbol{R})=(1 / N) \sum_{i} n\left(\boldsymbol{R}_{i}\right) n\left(\boldsymbol{R}_{i}+\boldsymbol{R}\right)$ and the density structure factor which is its FT. They have been presented in Fig. 2. We easily recognize the existence of charge modulations with the period of 3 lattice spacings in both directions. This 

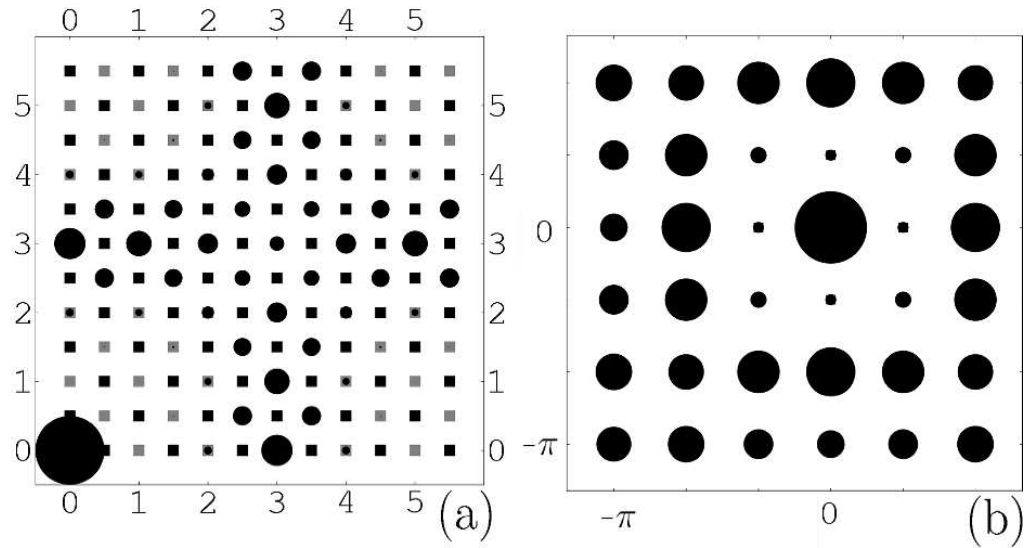

Fig. 2. Density correlation function (a) and density structure factor (b) of 4 HCBs on the 72 -site checkerboard-like cluster with periodic boundary conditions.

period is consistent with the picture of charges forming a square superlattice in the square lattice when there is a single charge per nine sites. This simple scenario is not true because the states corresponding to a system of charges forming a triangular lattice contribute to the ground state with high weights. Two examples of such states which minimize the Coulomb energy have been shown in Fig. 3.

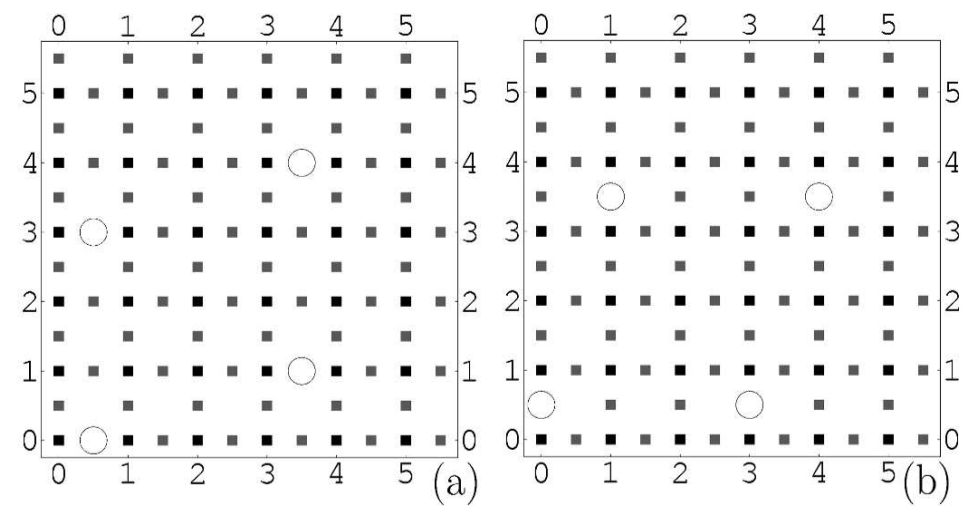

Fig. 3. Two states with highest weight in the ground-state.

In summary, in the first part of the paper we have discussed experimental data which indicate that checkerboard-like density modulations are formed in underdoped cuprates. Despite some serious indications based on STM measurements it seems that an alternative scenario of interference effects which give rise to some regularities in real space STM maps and their FTs cannot be ruled out. In the second part of the paper we have shown that ordering of local bound pairs, existing in underdoped cuprates due to attraction which originates in the local AF 
order, gives rise to regular charge modulations which have the full symmetry of the square lattice and thus resemble a checkerboard.

\section{Acknowledgments}

The author acknowledges partial support by the State Committee for Scientific Research under contract No. 2P03B00925.

\section{References}

[1] J.E. Hoffman, E.W. Hudson, K.M. Lang, V. Madhavan, H. Eisaki, S. Uchida, J.C. Davis, Science 295, 466 (2002).

[2] T. Hanaguri, C. Lupien, Y. Kohsaka, D.-H. Lee, M. Azuma, M. Takano, H. Takagi, J.C. Davis, Nature 430, 1001 (2004).

[3] J.E. Hoffman, K. McElroy, D.-H. Lee, K.M. Lang, H. Eisaki, S. Uchida, J.C. Davis, Science 297, 1148 (2002).

[4] M.R. Norman, H. Ding, M. Randeria, J.C. Campuzano, T. Yokoya, T. Takeuchi, T. Takahashi, T. Mochiku, K. Kadowaki, P. Guptasarma, D.G. Hinks, Nature 392, 157 (1998).

[5] T. Valla, A.V. Fedorov, P.D. Johnson, B.D. Wells, S.L. Hulbert, Q. Li, G.D. Gu, N. Koshizuka, Science 285, 2110 (1999).

[6] D.L. Feng, D.H. Lu, K.M. Shen, C. Kim, H. Eisaki, A. Damascelli, R. Yoshizaki, J.-I. Shimoyama, K. Kishio, G.D. Gu, S. Oh, A. Andrus, J. O’Donnell, J.N. Eckstein, Z.-X. Shen, Science 289, 277 (2000).

[7] K. McElroy, R.W. Simmonds, J.E. Hoffman, D.-H. Lee, J. Orenstein, H. Eisaki, S. Uchida, J.C. Davis, Nature 422, 592 (2003).

[8] K. McElroy, D.-H. Lee, J.E. Hoffman, K.M. Lang, J. Lee, E.W. Hudson, H. Eisaki, S. Uchida, J.C. Davis, Phys. Rev. Lett. 94, 197005 (2005).

[9] P. Wróbel, R. Eder, R. Micnas, J. Phys., Condens. Matter 15, 2755 (2003) and references therein. 